\title{
Analysis of quantum effects inside spherical charged black holes
}

\author{
Assaf Lanir, Amos Ori, Noa Zilberman, Orr Sela, Ahron Maline, and Adam Levi \\ Department of Physics, Technion, Haifa 32000, Israel
}

(Dated: May 4, 2021)

\begin{abstract}
We numerically compute the renormalized expectation value $\left\langle\hat{\Phi}^{2}\right\rangle_{\text {ren }}$ of a minimally-coupled massless quantum scalar field in the interior of a four-dimensional Reissner-Nordstrom black hole, in both the Hartle-Hawking and Unruh states. To this end we use a recently developed mode-sum renormalization scheme based on covariant point splitting. In both quantum states, $\left\langle\hat{\Phi}^{2}\right\rangle_{\text {ren }}$ is found to approach a finite value at the inner horizon (IH). The final approach to the IH asymptotic value is marked by an inverse-power tail $r_{*}^{-n}$, where $r_{*}$ is the Regge-Wheeler "tortoise coordinate", and with $n=2$ for the Hartle-Hawking state and $n=3$ for the Unruh state. We also report here the results of an analytical computation of these inverse-power tails of $\left\langle\hat{\Phi}^{2}\right\rangle_{\text {ren }}$ near the IH. Our numerical results show very good agreement with this analytical derivation (for both the power index and the tail amplitude), in both quantum states. Finally, from this asymptotic behavior of $\left\langle\hat{\Phi}^{2}\right\rangle_{\text {ren }}$ we analytically compute the leading-order asymptotic behavior of the trace $\left\langle\hat{T}_{\mu}^{\mu}\right\rangle_{\text {ren }}$ of the renormalized stress-energy tensor at the IH. In both quantum states this quantity is found to diverge like $b\left(r-r_{-}\right)^{-1} r_{*}^{-n-2}$ (with $n$ specified above, and with a known parameter $b$ ). To the best of our knowledge, this is the first fully-quantitative derivation of the asymptotic behavior of these renormalized quantities at the inner horizon of a four-dimensional Reissner-Nordstrom black hole.
\end{abstract}

Einstein's field equations admit black hole (BH) solutions endowed with remarkable exotic features including naked singularities, bridges to other universes and closed timelike curves. Among these solutions there is the Reissner-Nordstrom (RN) spacetime, describing a spherically-symmetric BH carrying electric charge. This spacetime metric is given by

$$
d s^{2}=-f(r) d t^{2}+\frac{1}{f(r)} d r^{2}+r^{2}\left(d \theta^{2}+\sin ^{2} \theta d \varphi^{2}\right),
$$

where $f(r)=1-2 M / r+Q^{2} / r^{2}, M$ and $Q$ being respectively the mass and charge of the BH. The event horizon $(\mathrm{EH})$ and the inner horizon (IH) are respectively located at $r=r_{+}$and $r=r_{-}$, the two solutions of $f(r)=0$ given by $r_{ \pm}=M \pm\left(M^{2}-Q^{2}\right)^{1 / 2}$. Interestingly, this metric may be analytically continued through the $\mathrm{BH}$ interior into a concatenation of asymptotically flat spacetime regions ('other universes'), accessible to an observer in the 'universe' where the $\mathrm{BH}$ originally formed only by traveling through the $\mathrm{BH}$. Along the way through the $\mathrm{BH}$ and into the 'other universes', the observer must cross the IH that lies inside the BH. It is a treacherous path, however, as classical perturbations appear to form a null curvature singularity along the Cauchy horizon $(\mathrm{CH}$; the ingoing section of the $\mathrm{IH}$ ). This is the situation in sphericallysymmetric charged $\mathrm{BHs}$ [1-6] as well as in spinning ones 77 9. Nevertheless, this null singularity, caused by classical perturbing fields, is known to be weak [10] (i.e. tidally nondestructive [11], with a $C^{0}$ limiting metric) — in both the charged [3] and spinning [7, 12] cases.

However, a general indication that emerges from a collection of analytical studies [13 15] on the effect of quantum perturbations inside $\mathrm{BHs}$ has been that semiclassical stress-energy fluxes are likely to diverge at the $\mathrm{CH}$, although so far it remained inconclusive in four dimensions. It is the goal of this work to address this issue via concrete numerical calculation (augmented by some analytical results) of the actual strength and form of these quantum effects inside a charged $\mathrm{BH}$.

Semiclassical gravity considers quantum matter fields propagating in a classical curved spacetime. The presence of curvature "deforms the vacuum" and induces a non-trivial stress energy in the quantum fields (even in "vacuum states"). In turn, this stress-energy tensor deforms the spacetime metric. This back-reaction effect is to be determined from the semiclassical Einstein's field equation

$$
G_{\mu \nu}=8 \pi\left\langle\hat{T}_{\mu \nu}\right\rangle_{r e n}
$$

Here $G_{\mu \nu}$ is the Einstein tensor of spacetime, and $\left\langle\hat{T}_{\mu \nu}\right\rangle_{\text {ren }}$ is the renormalized stress-energy tensor (RSET) associated with the quantum fields.

For simplicity, our choice for a quantum field is that of a minimally-coupled [33] massless scalar field, satisfying the massless Klein-Gordon equation $\square \hat{\Phi}=0$, where $\hat{\Phi}$ is the scalar field operator, and $\square$ denotes the covariant D'Alembertian. It proves useful to first compute the renormalized vacuum expectation value $\left\langle\hat{\Phi}^{2}\right\rangle_{\text {ren }}$ (often called the "vacuum polarization"), as it is simpler than the RSET, but still captures many of its essential features and provides important insight into the physical content of different vacua. Furthermore, as will be seen below, the behavior of $\left\langle\hat{\Phi}^{2}\right\rangle_{\text {ren }}$ actually determines the divergence rate of the RSET trace $\left\langle\hat{T}_{\mu}^{\mu}\right\rangle_{\text {ren }}$ at the IH.

Semiclassical gravity predicts the evaporation of $\mathrm{BHs}$ through the emission of Hawking radiation [16, 17]. BH evaporation obviously implies drastic differences in spacetime structure as compared to the corresponding classical picture. Likewise, it is conceivable that semiclassical stress-energy fluxes might affect the near- $\mathrm{CH}$ geometry inside RN (as well as Kerr) BHs more strongly than 
the classical perturbations do - potentially converting the $\mathrm{CH}$ into a strong (i.e. tidally destructive) spacelike singularity (and thereby preventing passage through the BH into the 'other universes'). However, these issues remained unresolved and to address them one must, obviously, compute the RSET in the interior region of BHs, and especially near the $\mathrm{CH}$. We have therefore set out to ultimately compute the RSET in BH interiors, and we present here novel results for a first step in this direction: the numerical computation of $\left\langle\hat{\Phi}^{2}\right\rangle_{\text {ren }}$ throughout the interior region 35] of a $\mathrm{RN} B H$ [34, followed by analysis of the leading-order behavior of $\left\langle\hat{\Phi}^{2}\right\rangle_{\text {ren }}$ and also $\left\langle\hat{T}_{\mu}^{\mu}\right\rangle_{\text {ren }}$ near the $\mathrm{CH}$.

The renormalization of the divergent $\left\langle\hat{\Phi}^{2}\right\rangle$ was carried out here by the recently developed pragmatic mode-sum (PMR) method [18, 19, which numerically implements the point-splitting renormalization scheme developed by Christensen [20, 21]. This prescription for $\left\langle\hat{\Phi}^{2}\right\rangle_{\text {ren }}$ (and the same concept holds for $\left\langle\hat{T}_{\mu \nu}\right\rangle_{r e n}$ as well) is depicted in the following equation:

$$
\left\langle\hat{\Phi}^{2}(x)\right\rangle_{r e n}=\lim _{x^{\prime} \rightarrow x}\left[\left\langle\hat{\Phi}(x) \hat{\Phi}\left(x^{\prime}\right)\right\rangle-G_{D S}\left(x, x^{\prime}\right)\right],
$$

where $G_{D S}\left(x, x^{\prime}\right)$ is the DeWitt-Schwinger counterterm (explicitly given in 22]).

We consider here $\left\langle\hat{\Phi}^{2}\right\rangle_{\text {ren }}$ in two quantum vacua. One is the Unruh state describing an evaporation of a $\mathrm{BH}$ [23], and the other is the Hartle-Hawking state $(\mathrm{HH})$ describing a $\mathrm{BH}$ in thermal equilibrium [24, 25] with an infinite bath of radiation. In Ref. 26] we derived an explicit expression for the scalar field two-point function in the RN interior, in both the Unruh and $\mathrm{HH}$ states, in terms of a radial function $\psi_{\omega l}(r)$ which can be computed numerically. This radial function satisfies the radial equation:

$$
\frac{d^{2} \psi_{\omega l}}{d r_{*}^{2}}+\left[\omega^{2}-V_{l}(r)\right] \psi_{\omega l}=0
$$

where $\omega$ denotes the mode's temporal frequency and $l$ its angular-momentum number. Here the effective potential $V_{l}(r)$ is given by

$$
V_{l}(r)=f(r)\left[\frac{l(l+1)}{r^{2}}+\frac{2 M}{r^{3}}-\frac{2 Q^{2}}{r^{4}}\right],
$$

and $r_{*}$ is the tortoise coordinate defined by $d r / d r_{*}=$ $f(r)$. Note that $r_{*} \rightarrow-\infty(+\infty)$ at the EH (IH). The boundary condition for $\psi_{\omega l}$ at the EH is

$$
\psi_{\omega l} \cong e^{-i \omega r_{*}}, \quad r_{*} \rightarrow-\infty
$$

The required input for the computation of $\left\langle\hat{\Phi}^{2}\right\rangle_{\text {ren }}$ inside the $\mathrm{BH}$ is the radial function $\psi_{\omega l}(r)$ and also $\rho_{\omega l}^{\mathrm{up}}$, namely the reflection coefficient for the "up" modes (see e.g. [26]) outside the BH. We compute $\psi_{\omega l}(r)$ and $\rho_{\omega l}^{\text {up }}$ numerically, and use them to construct the mode contributions to the two-point function inside the $\mathrm{BH}$, as prescribed in Ref. [26]. Then we regularize the mode sum using the $\theta$-splitting variant of our method, as described in [19]. This same method was implemented recently for computing $\left\langle\hat{\Phi}^{2}\right\rangle_{\text {ren }}$ inside a Schwarzschild BH in Ref. 27, where a more detailed account of the procedure is provided. (Additional details are provided in the Supplementary materials.)

From the symmetries of the RN geometry it immediately follows that $\left\langle\hat{\Phi}^{2}\right\rangle_{\text {ren }}$ (like $\left\langle\hat{T}_{\mu}^{\mu}\right\rangle_{\text {ren }}$ ) only depends on $r$. In the next section we present the results for $\left\langle\hat{\Phi}^{2}(r)\right\rangle_{\text {ren }}$ throughout the range $r_{-} \leq r \leq r_{+}$. Interestingly, it turns out that for both the Unruh and HH states, $\left\langle\hat{\Phi}^{2}\right\rangle_{\text {ren }}$ remains finite upon approaching the IH (although its gradient diverges there). Then subsequently we present analytical results for the asymptotic behaviors of $\left\langle\hat{\Phi}^{2}\right\rangle_{\text {ren }}$ and $\left\langle\hat{T}_{\mu}^{\mu}\right\rangle_{\text {ren }}$ very close to the IH, and for $\left\langle\hat{\Phi}^{2}\right\rangle_{\text {ren }}$ we also compare our analytical and numerical results.

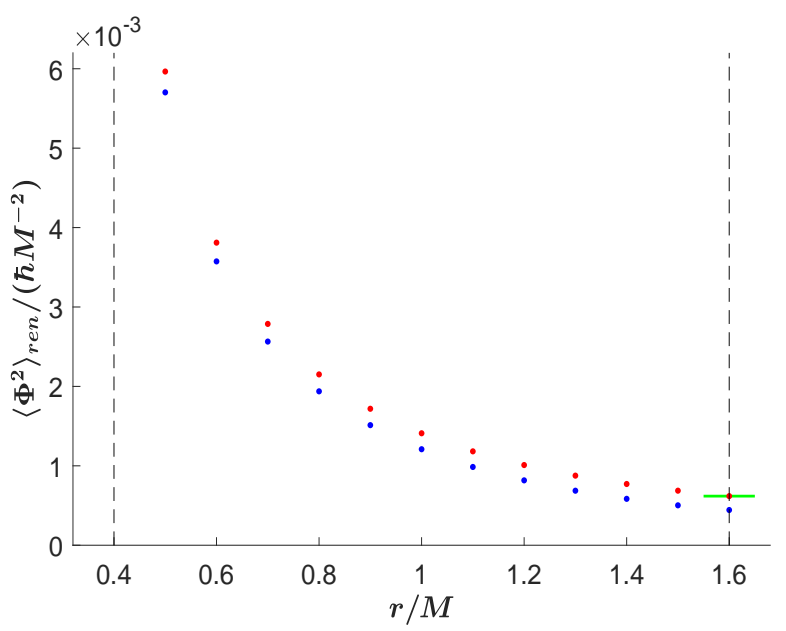

Figure 1. The numerically computed $\left\langle\hat{\Phi}^{2}(r)\right\rangle_{\text {ren }}$ in the HH (red) and Unruh (blue) states in the region between the two horizons. The short horizontal green line represents the analytical result for $\left\langle\hat{\Phi}^{2}(r)\right\rangle_{r e n}$ in the HH state at the EH.

Numerical results: We shall focus here on the specific example $Q / M=0.8$. In this case $r_{+}=1.6 M$ and $r_{-}=$ $0.4 M$. The radial equation (4) together with the initial condition (6) was solved numerically for $\psi_{\omega l}(r)$, from the EH to very close to the IH, for a sufficiently dense set of $\omega l$ modes in the range $0 \leq l \leq 10$ and $0<\omega<10 / M$. The reflection coefficient $\rho_{\omega l}^{\mathrm{up}}$ was also computed numerically for these $\omega l$ modes. These quantities were then used to construct $\left\langle\hat{\Phi}^{2}\right\rangle_{\text {ren }}$. See supplementary materials for more details.

Figure 1 displays the numerical results for $\left\langle\hat{\Phi}^{2}(r)\right\rangle_{r e n}$ in the region between the two horizons (specifically for $0.5 \leq r / M \leq 1.6$ ), for both quantum states. Our result for the $\mathrm{HH}$ state agrees very nicely with the known analytical result [28, 29] at the $\mathrm{EH}$, with a difference of only 
$\sim 0.005 \%$.

The most obvious feature seen in this figure is the steady growth with decreasing $r$, which becomes steeper when getting close to the IH. This trend of sharp increase towards the IH continues all the way up to, say, $r-r_{-} \sim 10^{-6} M$. From this behavior one might get the impression (as we originally did) that $\left\langle\hat{\Phi}^{2}(r)\right\rangle_{\text {ren }}$ would diverge at the $\mathrm{IH}$.

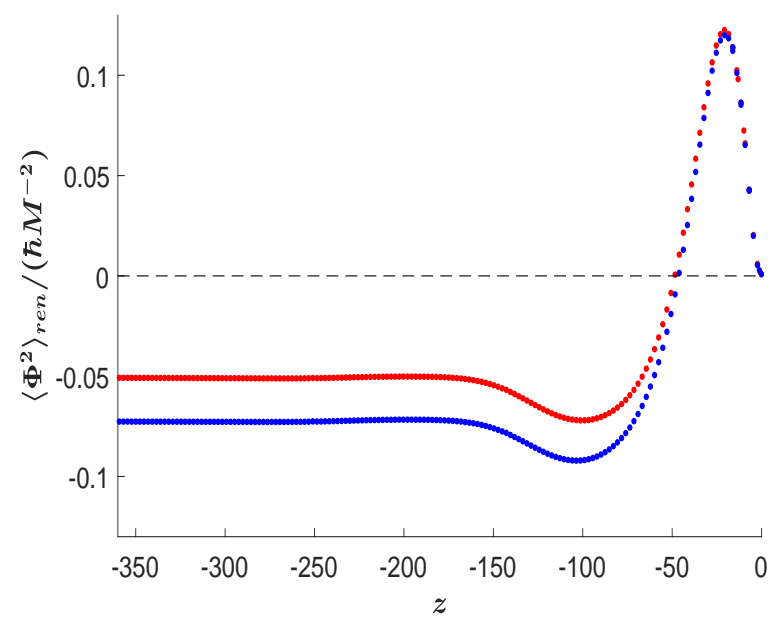

Figure 2. $\left\langle\hat{\Phi}^{2}(r)\right\rangle_{\text {ren }}$ in the HH (red) and Unruh (blue) states, as a function of $z$.

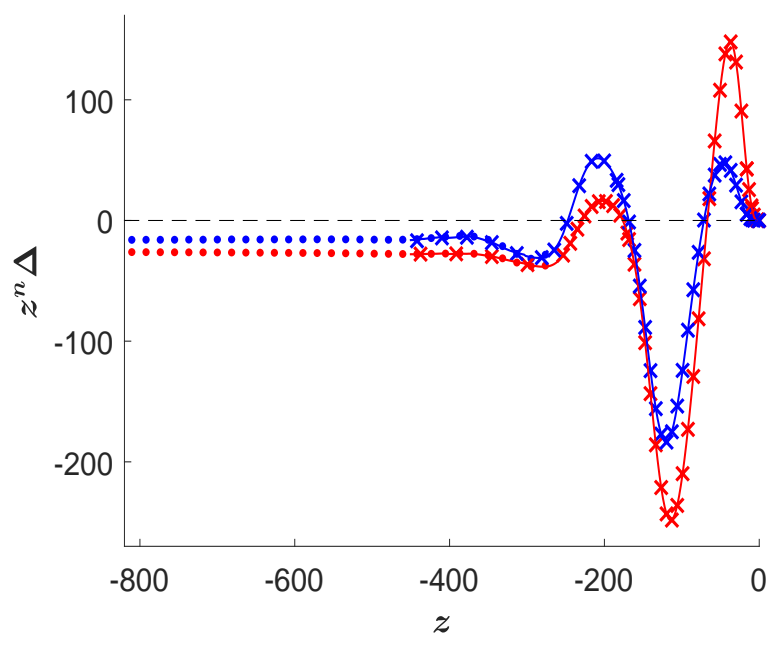

Figure 3. $\Delta(z) \cdot z^{n}$ in the $\mathrm{HH}$ (red) and Unruh (blue) states, in the region $-845<z<0$ (which roughly corresponds to $\left.10^{-367}<\delta r<1\right)$. The results for the Unruh state are divided here by a factor of -150 , for convenience. The plateaus at the left half of the $z$ axis indicate the inverse-power behavior $\Delta \propto z^{-n}$. For both the HH and Unruh results, the crosses indicate full numerical results, the solid curves indicate semiasymptotic results, and the dots indicate the "refined variant" results.

To our surprise, we found that this picture drastically changes once we start exploring regions much closer to the IH. In fact, $\left\langle\hat{\Phi}^{2}(r)\right\rangle_{\text {ren }}$ eventually approaches a finite value at $r \rightarrow r_{-}$, which we denote by $\left(\hbar / M^{2}\right)\left\langle\hat{\Phi}^{2}\right\rangle_{-}$, where the index "-" refers to the limit $r \rightarrow r_{-}$. This is clearly seen in Fig. 2, which displays $\left\langle\hat{\Phi}^{2}\right\rangle_{\text {ren }}$ as a function of the logarithmic variable $z$ defined by

$$
z \equiv \ln (\delta r), \quad \delta r \equiv\left(r-r_{-}\right) / M .
$$

Note that the IH corresponds to $z \rightarrow-\infty$. In both quantum states, after a few quickly-decaying oscillations (there are actually two maxima and two minima overall, although not all of them can be seen in this figure), $\left\langle\hat{\Phi}^{2}\right\rangle_{\text {ren }}$ approaches a plateau. The asymptotic values are $\left\langle\hat{\Phi}^{2}\right\rangle_{-}^{H} \cong-0.05058$ and $\left\langle\hat{\Phi}^{2}\right\rangle_{-}^{U} \cong-0.07258$. Hereafter, an index "H" or "U" will denote the HH state or Unruh state, respectively.

Near-IH asymptotic behavior: To explore the near$\mathrm{IH}$ asymptotic behavior we define (respectively for each quantum state)

$$
\Delta \equiv\left(M^{2} / \hbar\right)\left\langle\hat{\Phi}^{2}\right\rangle_{r e n}-\left\langle\hat{\Phi}^{2}\right\rangle_{-}
$$

(i.e. the dimensionless deviation from $\left\langle\hat{\Phi}^{2}\right\rangle_{-}$).

As it turns out, $\Delta(z)$ decays like $z^{-n}$, where hereafter $n$ will stand for either $n_{H}=2$ (HH state) or $n_{U}=3$ (Unruh state). To demonstrate this, Fig. 3 displays $z^{n} \cdot \Delta(z)$. The flat horizontal forms of the red and blue lines, at the left half of the $z$ axis, clearly indicate this leading-order behavior $\Delta \propto z^{-n}$ in the two quantum states. (This behavior is seen even more clearly in Fig. 4.)

The effective potential $V_{l}(r)$, given in (5), vanishes at the IH like $f \propto \delta r$. Therefore, for sufficiently small $\delta r$ the radial equation (4) becomes free, and its general solution in that domain is

$$
\psi_{\omega l} \cong A_{\omega l} e^{i \omega r_{*}}+B_{\omega l} e^{-i \omega r_{*}} . \quad(\delta r \ll 1)
$$

The coefficients $A_{\omega l}, B_{\omega l}$ are dictated by the scattering problem off the potential $V_{l}(r)$ from the EH to the $\mathrm{IH}$, and can be determined numerically. Note that on approaching the IH $r_{*}$ diverges as $r_{*} \approx-z / 2 \kappa_{-}$, where $\kappa_{-}=\left(r_{+}-r_{-}\right) / 2 r_{-}^{2}$ is the IH surface gravity.

In order to explore the aforementioned inverse-power decay we need to push the numerical solution to extremely small $\delta r$ values, say $\delta r<e^{-400} \sim 10^{-175}$, as can be seen in e.g. Fig. 3. This is hard to do with the brute-force numerical solution for $\psi_{\omega l}$. [One of the difficulties, already seen in Eq. (9), is the very rapid variation of $\psi_{\omega l}$ with $\omega$ for $r_{*} \gg 1$.] To overcome this difficulty, we introduce the semi-asymptotic approximation, in which we simply employ Eq. (9) as an approximation to $\psi_{\omega l}$ for sufficiently small $\delta r$. The results obtained from this approximation are displayed in Fig. 3 by the red and blue solid curves.

Still, in the deep tails region (say $z<-700$ ) even this semi-asymptotic approximation starts to be noisy (when 
numerically implemented to explore the inverse-power tails). We therefore designed a refined variant of this approximation, aimed to explore the tails region, which can more efficiently take us to very large $|z|$ values. It is this refined variant that we have used to produce Fig. 4 below (and also the left region in Fig. 3). We point out that there are nice overlap regions on the $z$ axis between these three slightly different numerical procedures, as may be seen e.g. in Fig. 3. This is further discussed in the Supplementary materials, which provide additional information about the semi-asymptotic approximation and its refined variant.

Analytical expressions for the inverse-power tails: To our pleasant surprise, we found that it is possible to obtain, analytically [30, the dominant inverse-power tails characterizing the near-IH asymptotic behavior of $\Delta(z)$. This is possible because, as it turns out, these tails are actually governed by the small- $\omega$ asymptotic behavior of $A_{\omega l}, B_{\omega l}$, and $\rho_{\omega l}^{\text {up }}$; and this small- $\omega$ behavior can be deduced analytically. This analysis yields the two dominant inverse powers $\left(n_{H}=2\right.$ and $\left.n_{U}=3\right)$ as well as their multiplicative amplitude parameters (for both quantum states).

Furthermore, since we had to carry the analysis to or$\operatorname{der} z^{-3}$ (needed for the Unruh-state leading order), we actually got, almost for free, the term $\propto z^{-3}$ for the $\mathrm{HH}$ state as well. Thus, including all the inverse-power terms to which we presently have analytical access, we write the tail expressions as

$$
\Delta_{U}=C_{U} z^{-3}+\ldots, \Delta_{H}=C_{H} z^{-2}+C_{H}^{1} z^{-3}+\ldots,
$$

where "..." denotes higher-order corrections. Defining $\alpha \equiv r_{+} / r_{-}$, we find

$$
\begin{gathered}
C_{U}=2 \Lambda\left(1-\alpha^{4}\right)(1-\alpha)^{2}\left(11+14 \alpha+11 \alpha^{2}\right) \\
C_{H}=3 \Lambda \alpha^{-2}\left(1-\alpha^{4}\right)^{2} \\
C_{H}^{1}=2 \log \left[\frac{2(\alpha-1)}{\alpha+1}\right] C_{H}-\frac{1}{4}\left(\alpha^{-2}-3\right) C_{U}
\end{gathered}
$$

where $\Lambda \equiv\left(1-\alpha^{2}\right) / 768 \pi^{2}$.

Figure 4 displays the analytical expressions 10 (black curves) and the numerical data (dots) for the inversepower tails, for both quantum states, in the range $400<$ $-z<1500$. It shows excellent agreement, supporting the validity/accuracy of both the theoretical analysis and numerics.

Trace of the stress tensor: For a minimally coupled massless scalar field, the RSET trace $\left\langle\hat{T}_{\mu}^{\mu}\right\rangle_{\text {ren }}$ is uniquely determined [26] by $\left\langle\hat{\Phi}^{2}(x)\right\rangle_{\text {ren }}$ via

$$
\left\langle\hat{T}_{\mu}^{\mu}\right\rangle_{\text {ren }}=-\frac{1}{2} \square\left\langle\hat{\Phi}^{2}(x)\right\rangle_{\text {ren }}+\text { (local term) }
$$

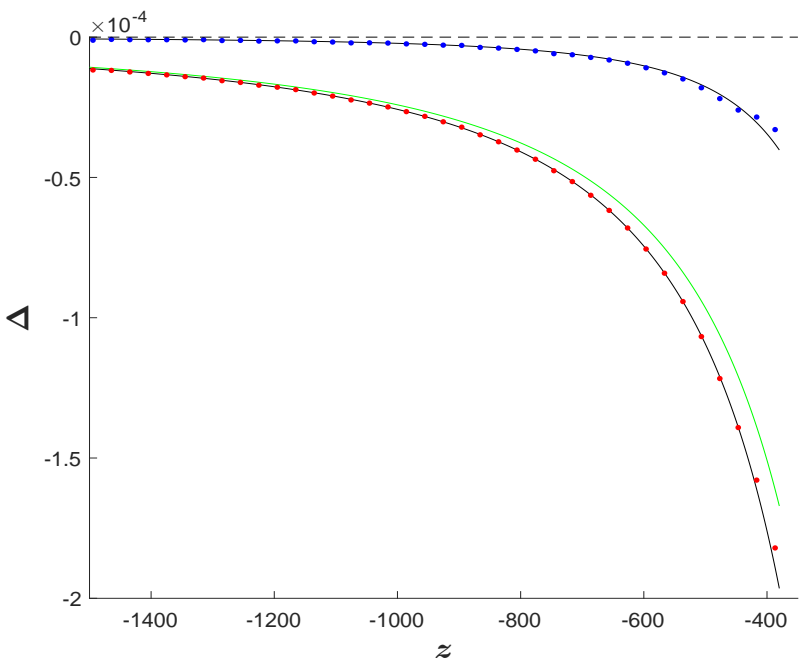

Figure 4. $\Delta(z)$ in the HH state (red dots) and Unruh state (blue dots) exceedingly close to $r_{-}$(up to $z \sim 1500$, which roughly corresponds to $\delta r \sim 10^{-650}$ ), computed using the "refined variant". The black curves are the analytical expressions 10 for the inverse-power tails. The green curve indicates the leading-order analytical result $\Delta_{H} \approx C_{H} z^{-2}$ (whereas the corresponding black curve also includes the next-order term $\left.C_{H}^{1} z^{-3}\right)$.

The local term only depends on the background metric, which is perfectly regular at the IH. Therefore the singular piece of $\left\langle\hat{T}_{\mu}^{\mu}\right\rangle_{\text {ren }}$ is fully described by the D'Alembertian term. Since the constant $\left\langle\hat{\Phi}^{2}\right\rangle_{-}$contributes nothing to the D'Alembertian, we are left with $-\left(\hbar / 2 M^{2}\right) \square \Delta$. Applying the D'Alembertian operator to Eq. $(10)$, we obtain for the two quantum states, at leading order in $1 / z($ and $\delta r)$ :

$$
\left\langle\hat{T}_{\mu}^{\mu}\right\rangle_{\text {ren }} \cong n(n+1) \frac{\hbar}{M^{2}} \kappa_{-} \frac{C}{r-r_{-}} z^{-n-2},
$$

where, recall, $n_{H}=2, n_{U}=3$ and $C$ is either $C_{H}$ or $C_{U}$ specified above.

Discussion: We found that $\left\langle\hat{\Phi}^{2}\right\rangle_{\text {ren }}$ is finite at the IH. This finite asymptotic value is approached via a few quickly decaying oscillations followed by an inverse-power tail. In turn, the RSET trace $\left\langle\hat{T}_{\mu}^{\mu}\right\rangle_{r e n}$ diverges as $1 /(r-$ $\left.r_{-}\right)$softened by a certain inverse power of $\ln \left(r-r_{-}\right)$. We obtained a fully analytical description of this divergent trace (at leading order), Eq. (15.

Here we only investigated numerically the case $Q / M=$ 0.8. However, our results for the inverse power tails and, more importantly, for the asymptotic divergence 15) of the RSET trace - apply to any (non-extremal) $M$ and $Q$.

The behavior of $\left\langle\hat{\Phi}^{2}\right\rangle_{\text {ren }}$ on approaching the IH is remarkably complex. In particular, the final inverse-power tails are only exposed at, say, $\delta r<10^{-175}$. This complex asymptotic behavior may be traced to the factors $e^{ \pm i \omega r_{*}}$ in Eq. (9). The mode contribution to $\left\langle\hat{\Phi}^{2}\right\rangle_{\text {ren }}$ contains 
terms quadratic in $\psi_{\omega l}$, including factors $e^{ \pm 2 i \omega r_{*}}$ ( multiplying certain functions of $A(\omega), B(\omega)$, etc.). Integration over $\omega$ then leaves a nontrivial function of $r_{*}$, embodied in the asymptotic behavior of $\left\langle\hat{\Phi}^{2}\right\rangle_{\text {ren }}$.

It is interesting to compare these results to a recent work 31 carried out by one of us (OS), in which the large- $l$ approximation was used to obtain bounds on the divergence rate of $\left\langle\hat{\Phi}^{2}\right\rangle_{\text {ren }},\left\langle\hat{T}_{\mu}^{\mu}\right\rangle_{\text {ren }}$, and certain components of $\left\langle\hat{T}_{\mu \nu}\right\rangle_{\text {ren }}$. In particular it was found that for both the Unruh and HH states $\left\langle\hat{\Phi}^{2}\right\rangle_{\text {ren }}$ and $\left\langle\hat{T}_{\mu}^{\mu}\right\rangle_{\text {ren }}$ must be less divergent than $1 /\left(r-r_{-}\right)$and $1 /\left(r-r_{-}\right)^{2}$, respectively. The results presented here for these two quantities are fully consistent with these bounds.

The expressions presented here for the pre-factors $C_{H}, C_{U}$ that control the divergence of $\left\langle\hat{T}_{\mu}^{\mu}\right\rangle_{\text {ren }}$, only apply to a minimally-coupled massless scalar field. In the case of non-minimal coupling they will change. In particular, in the case of conformal coupling these prefactors will vanish altogether, because the standard traceanomaly formula guarantees regularity of the trace at the IH. The same situation will occur in the case of a quantum electromagnetic field, since this field is conformal too.

It is still unclear, however, if the gravitational semiclassical contribution to the effective stress-energy will possess such a trace divergence at the IH. The presence of a gravitational contribution (associated with quantized linearized modes of the gravitational field) to the effective $\left\langle\hat{T}_{\mu \nu}\right\rangle_{\text {ren }}$ is obvious from the very basic fact that gravitons do significantly contribute to Hawking radiation [32] (and correspondingly, negative semiclassical gravitational-field influx must penetrate into the $\mathrm{EH}$ of the evaporating $\mathrm{BH}$ and contribute to its shrinkage). However, a formalism for quantifying the semiclassical effective gravitational stress-energy tensor has not been formulated so far.

This analysis calls for extension in several obvious directions. The first obvious step is to elevate the analysis from $\left\langle\hat{\Phi}^{2}\right\rangle_{\text {ren }}$ to the RSET. Second, the quantum scalar field should better be replaced by the (more realistic) quantum electromagnetic field. In addition, it will be important to extend the analysis from RN to the Kerr background (a spinning BH), which is obviously much more realistic than a spherical charged $\mathrm{BH}$.

Finally, it will be very interesting (but also very challenging) to explore the back-reaction effect of the semiclassical RSET on the BH interior, according to the semiclassical Einstein equation (2).

We thank Robert Wald, Marc Casals, and Adrian Ottewill for interesting and helpful discussions. This research was supported by the Asher Fund for Space Research at the Technion. The work of AL and OS was further supported by the Israel Science Foundation under Grant No. 1696/15 and by the I-CORE Program of the Planning and Budgeting Committee. The work of
NZ was partly supported by the Israel Science Foundation under Grant No. 600/18.

[1] W. A. Hiscock, Evolution of the interior of a charged black hole, Phys. Rev. Lett. 83, 110 (1981).

[2] E. Poisson and W. Israel, Internal structure of black holes, Phys. Rev. D. 41, 1796 (1990).

[3] A. Ori, Inner structure of a charged black hole: An exact mass-inflation solution, Phys. Rev. Lett. 67, 789 (1991).

[4] P. R. Brady and J. D. Smith, Black hole singularities: a numerical approach, Phys. Rev. Lett. 75, 1256 (1995).

[5] S. Hod and T. Piran, Mass Inflation in Dynamical Gravitational Collapse of a Charged Scalar Field, Phys. Rev. Lett. 81, 1554 (1998).

[6] L. M. Burko, Structure of the black hole's Cauchyhorizon singularity, Phys. Rev. Lett. 79, 4958 (1997).

[7] A. Ori, Structure of the singularity inside a realistic rotating black hole, Phys. Rev. Lett. 68, 2117 (1992).

[8] P. R. Brady, S. Droz, and S. M. Morsnik, Late-time singularity inside nonspherical black holes, Phys. Rev. D. 58, 084034 (1998).

[9] A. Ori, Oscillatory null singularity inside realistic spinning black holes, Phys. Rev. Lett. 83, 2117 (1999).

[10] F. J. Tipler, Singularities in conformally flat spacetimes, Phys. Lett. A 64, 8 (1977).

[11] A. Ori, Strength of curvature singularities, Phys. Rev. D 61, 064016 (2000).

[12] M. Dafermos and J. Luk, The interior of dynamical vacuum black holes I: The $C^{0}$-stability of the Kerr Cauchy horizon, arXiv:1710.01722 [gr-qc]] (2017).

[13] N. D. Birrell and P. C. W. Davies, On falling through a black hole into another universe, Nature (London) 272, 35 (1978).

[14] A. C. Ottewill and E. Winstanley, Renormalized stress tensor in Kerr space-time: General results, Phys. Rev. D. 62, 084018 (2000).

[15] W. A. Hiscock, Quantum-mechanical instability of the Kerr-Newman black-hole interior, Phys. Rev. D. 21, 2057 (1980).

[16] S. W. Hawking, Black hole explosions?, Nature 248, 30 (1974).

[17] S. W. Hawking, Particle creation by black holes, Commun. Math. Phys. 43, 199 (1975).

[18] A. Levi and A. Ori, Pragmatic mode-sum regularization method for semiclassical black-hole spacetimes, Phys. Rev. D. 91, 104028 (2015).

[19] A. Levi and A. Ori, Mode-sum regularization of $\left\langle\phi^{2}\right\rangle$ in the angular-splitting method, Phys. Rev. D. 94, 044054 (2016).

[20] S. M. Christensen, Vacuum expectation value of the stress tensor in an arbitrary curved background: The covariant point separation method, Phys. Rev. D. 14, 2490 (1976).

[21] S. M. Christensen, Regularization, renormalization, and covariant geodesic point separation, Phys. Rev. D. 17, 946 (1978).

[22] P. R. Anderson, W. A. Hiscock and D. A. Samuel, Stressenergy tensor of quantized scalar fields in static spherically symmetric spacetimes, Phys. Rev. D. 51, 4337 (1995). 
[23] W. G. Unruh Notes on black-hole evaporation, Phys. Rev. D. 14, 870 (1976).

[24] J. B. Hartle and S. W. Hawking Path-integral derivation of black-hole radiance, Phys. Rev. D. 13, 2188 (1976).

[25] W. Israel Thermo-field dynamics of black holes, Phys. Lett. A. 57, 107 (1976).

[26] A. Lanir, A. Levi, A. Ori and O. Sela Two-point function of a quantum scalar field in the interior region of a Reissner-Nordstrom black hole, Phys. Rev. D. 97, 024033 (2018).

[27] A. Lanir, A. Levi, and A. Ori, Mode-sum renormalization of $\left\langle\hat{\Phi}^{2}\right\rangle$ for a quantum scalar field inside a Schwarzschild black hole, Phys. Rev. D. 98, 084017 (2018).

[28] V. P. Frolov, Vacuum polarization near the event horizon of a charged rotating black hole, Phys. Rev. D. 26, 954 (1982).

[29] A. Ottewill, private communication.

[30] A. Maline et al (in preparation).

[31] O. Sela, Quantum effects near the Cauchy horizon of a Reissner-Nordstrom black hole, Phys. Rev. D. 98, 024025 (2018).

[32] D. N. Page, Particle emission rates from a black hole: Massless particles from an uncharged, nonrotating hole, Phys. Rev. D. 13, 198 (1976).

[33] The results described below for $\left\langle\hat{\Phi}^{2}\right\rangle_{\text {ren }}$ are actually independent of the coupling constant (because the Ricci scalar vanishes in our case). However, the results for $\left\langle\hat{T}_{\mu}^{\mu}\right\rangle_{\text {ren }}$ only apply for a minimally-coupled field.

[34] We shall consider here a fixed RN geometry, without back-reaction.

[35] Throughout, by "interior region" we actually refer to the 'predictable' internal domain $r_{-} \leq r \leq r_{+}$.

\section{SUPPLEMENTAL MATERIAL}

\section{A. Method of computation of $\left\langle\hat{\Phi}^{2}\right\rangle_{\text {ren }}$}

We use here the mode-sum expression derived in Ref. [26] for the TPF, to which we apply the $\theta$-splitting variant [19] of the PMR method. In this treatment, we follow the same procedure used in Sec. 3 of Ref. [27], where a step-by-step recipe for the computation of $\left\langle\hat{\Phi}^{2}\right\rangle_{\text {ren }}$ inside a Schwarzschild BH is provided. Here we use exactly the same procedure except for the following trivial modifications (which follow directly from the change of the metric from Schwarzschild to $\mathrm{RN}$ ): (i) The $\mathrm{EH}$ surface gravity, $\kappa$ of Ref. [27] , is here replaced by $\kappa_{+} \equiv\left(r_{+}-r_{-}\right) / 2 r_{+}^{2}$. (ii) $d(r)$ of Eq. (3.11) therein is here replaced by $d(r)=\left(Q^{2}-M r\right) / 24 \pi^{2} r^{4}$. (iii) The counter-term $G_{D S}\left(x, x^{\prime}\right)$, described in Eq. (3.3) therein for the Schwarzschild case, now has a (finite) additional term proportional to the Ricci tensor. Note, however, that this change is already incorporated in the aforementioned change in $d(r)$. Therefore, in the operational part of Sec. 3 of Ref. [27] only changes (i) and (ii) need be considered.

\section{B. Numerical parameters}

In the purpose of computing $\left\langle\hat{\Phi}^{2}\right\rangle_{\text {ren }}$ in the vicinity of the IH, the radial equation was solved for $11 l$ values $(0 \leq l \leq 10)$, and for each $l$ in the range $\omega \in$ $\left[0,10 M^{-1}\right]$ with uniform spacings of $d \omega=0.0005 M^{-1}$ and $d \omega=0.004 M^{-1}$ in the regions $\omega \in\left[0,2 M^{-1}\right]$ and $\omega \in\left[2 M^{-1}, 10 M^{-1}\right]$, respectively. The computation of $\left\langle\hat{\Phi}^{2}\right\rangle_{\text {ren }}$ in the general region between the $\mathrm{EH}$ and IH (namely $0.5 \leq r \leq 1.6$ ) involved solving the radial equation for $31 l$ values $(0 \leq l \leq 30)$, and for each $l$ in the range $\omega \in\left[0,120 M^{-1}\right]$ with uniform spacings of $d \omega=0.0005 M^{-1}$ and $d \omega=0.1 M^{-1}$ in the regions $\omega \in\left[0,6 M^{-1}\right]$ and $\omega \in\left[6 M^{-1}, 120 M^{-1}\right]$, respectively. For the computation in the region close to the $\mathrm{EH}$, the radial equation was solved for $16 l$ values $(0 \leq l \leq 15)$, and for each $l$ in the range $\omega \in\left[0,15 M^{-1}\right]$ with uniform spacings of $d \omega=0.01 M^{-1}$.

\section{The semi-asymptotic approximation and its refined variant}

A key ingredient in the computation of $\left\langle\hat{\Phi}^{2}\right\rangle_{\text {ren }}$ inside the $\mathrm{BH}$ is the quantity $E_{\omega l}(r)$, given in Eqs. $(3.7,3.8)$ of Ref. [27] (for the Unruh and HH states respectively). It involves the function $\bar{\psi}_{\omega l}$ which is specified in Eq. (3.5) therein in terms of the radial function $\psi_{\omega l}$.

In the semi-asymptotic approximation we leave $E_{\omega l}(r)$ unchanged except that we substitute the near-IH asymptotic expression (9) for $\psi_{\omega l}$, and also substitute $r_{-}$for $r$. For the HH state we get:

$$
E_{\omega l}^{H}=\frac{1}{2 \pi|\omega| r_{-}^{2}} \operatorname{Re}\left[\left(\left|A_{\omega l}\right|^{2}+\left|B_{\omega l}\right|^{2}+2 A_{\omega l} B_{\omega l}^{*} e^{2 i \omega r_{*}}\right) \operatorname{coth} \tilde{\omega}+\frac{\rho_{\omega l}^{\mathrm{up}}}{\sinh \tilde{\omega}}\left(A_{\omega l}^{2} e^{2 i \omega r_{*}}+B_{\omega l}^{2} e^{-2 i \omega r_{*}}+2 A_{\omega l} B_{\omega l}\right)\right]
$$

where $\tilde{\omega} \equiv \pi \omega / \kappa_{+}$. In principle, this quantity needs be subsequently integrated over $\omega$ (and also summed over $l$ ). Note, however, that at large $\omega$ both $A_{\omega l}$ and $\rho_{\omega l}^{\text {up }}$ decay exponentially while $B_{\omega l} \rightarrow 1$, hence $E_{\omega l}^{H} \propto \omega^{-1}$ and its integral over $\omega$ diverges. To this end, in the original $\theta$ - splitting method we subtract $E_{\omega, l=0}$ from $E_{\omega l}$ before integration, see e.g. Eq. (3.9) therein. This $l=0$ subtraction is also done in the semi-asymptotic approximation.

In the refined variant we proceed in a different manner. From the squared brackets in Eq. (16) we subtract two 
terms:

$$
\delta E_{1} \equiv\left(\left|A_{\omega l}\right|^{2}+\left|B_{\omega l}\right|^{2}\right) \operatorname{coth} \tilde{\omega}+\frac{2 \rho_{\omega l}^{\mathrm{up}}}{\sinh \tilde{\omega}} A_{\omega l} B_{\omega l}
$$

and

$$
\delta E_{2} \equiv \frac{1}{\sinh \tilde{\omega}}\left(2 A_{0} B_{0}^{*}-A_{0}^{2} e^{2 i \omega r_{*}}-B_{0}^{2} e^{-2 i \omega r_{*}}\right),
$$

where $A_{0}$ and $B_{0}$ denote the $\omega \rightarrow 0$ limits of $A$ and $B$ respectively. [These parameters can actually be obtained analytically, they are given by $(-1)^{l}\left(r_{-}^{2} \pm r_{+}^{2}\right) / 2 r_{+} r_{-}$, with the + sign for $B_{0}$ and the - sign for $A_{0}$, but we shall not discuss this derivation here.] The subtraction of $\delta E_{1}$ removes the $\propto \omega^{-1}$ term at large $\omega$. In turn, the subtraction of $\delta E_{2}$ removes the $\omega \rightarrow 0$ divergence that would have been caused by the $\delta E_{1}$ subtraction. We denote the modified $E_{\omega l}^{H}$ (due to this subtraction) by $\hat{E}_{\omega l}^{H}$ for later convenience.

Obviously, this subtraction of $\delta E_{1}$ and $\delta E_{2}$ needs to be justified. The subtraction of $\delta E_{2}$ is allowed because it is independent of $l$, and adding any $l$-independent term to the mode contribution $E_{\omega l}$ does not affect the resultant $\left\langle\hat{\Phi}^{2}\right\rangle_{\text {ren }}$ at all (it just modifies the "blind spot" [19]).

The other term $\delta E_{1}$ does depend on $l$, but nevertheless it is independent of $r_{*}$. As a consequence, although $\left\langle\hat{\Phi}^{2}\right\rangle_{\text {ren }}$ is changed by this subtraction, this change merely amounts to adding some $r$-independent constant to $\left\langle\hat{\Phi}^{2}\right\rangle_{\text {ren }}$. Therefore, this subtraction does not affect $\Delta(z)$ at all. We can thus use the refined version in e.g. Figs. 3 and 4 , which describe $\Delta(z)$ (but not in Figs. 1 and 2 that describe the full $\left\langle\hat{\Phi}^{2}\right\rangle_{\text {ren }}$; see also last paragraph).

Since the subtraction of $\delta E_{1}$ removes the large- $\omega$ irregularity, we no longer need to subtract the $l=0$ contribution. Thus, in Eq. (3.9) of Ref. [27] the integrand (i.e. the term in squared bracket) is now simply replaced by $\hat{E}_{\omega l}^{H}$. This new integrand actually decays (at large $\omega$ ) exponentially in $\omega$, leading to a quick and efficient numerical convergence of the integral. We denote the resultant integral by $\hat{F}(l, r)$.

We find, somewhat surprisingly, that the quantity $\hat{F}_{\text {reg }}(l, r)=\hat{F}(l, r)-F_{\text {sing }}(l, r)$ decays to zero at large $l$ (i.e. there is no blind spot). Therefore, in the refined variant there is no need to define the function $H$ [Eq. (3.14) therein]. Instead, the final result is simply obtained (up to some $r$-independent shift) by summing $[(2 l+1) /(4 \pi)] \hat{F}_{\text {reg }}(l, r)$ over $l$.

Furthermore, at large $-z$ this sum over $l$ converges tremendously fast. For example, the numerical data presented in Fig. 4 were obtained by summing over $l$ up to $l=2$. But in fact, the contribution from $l=0$ looks just the same; the contribution from $l=1$ cannot be seen in the graph (let alone the $l=2$ contribution). Although unnecessary, we chose to include the $l=1$ and $l=2$ terms as well in our computation.

So far we focused on the $\mathrm{HH}$ state for concreteness. The refined computation of $\Delta$ in the Unruh state may proceed in a fully analogous manner. In both quantum states, the exponential decay of the integrand with $\omega$, combined with the extremely fast convergence of the sum of $\hat{F}_{\text {reg }}(l)$ over $l$, lead to numerical results of a considerably better quality, which is the purpose we sought to fulfill when we employed the refined method.

Finally, we use this opportunity to clarify again which of the three variants was used in each of the figures. Both Figs. 1 and 2 were produced by the full-fledged computational scheme. In contrast, Fig. 4 displays the refined results for $\Delta(z)$. Figure 3 , which presents $z^{n} \cdot \Delta(z)$, displays the results obtained from all three variants. Notice the ranges of overlaps between these different variants: The full-fledged numerics and the semiasymptotic approximation overlap in the very wide domain $-450<z<-3$. In addition, all three variants overlap in the range $-450<z<-280$. This figure demonstrates nice agreement between the different variants throughout the domains of their overlap. 